\title{
NOTES MORPHOLOGIQUES
}

\section{SUR LES TRYPANOSOMOSES ANIMALES DE STANLEYVILLE}

\section{ET DU CONGO ORIENTAL}

\author{
Par J. SCHWETZ
}

Du mois d'août 1927 jusqu'au premier janvier 1930, une grande partie de l'activité du Laboratoire de Stanleyville a été consacrée à la trypanosomose animale, à l'examen et au réexamen des divers troupeaux et au traitement des bêtes trypanosées. Nous étions aidés dans ce travail, jusqu'à la fin de 1928, par notre assistante Mlle Geerinck. A partir de cette époque, la grosse besogne de la trypanosomose animale était assurée par M. Storck, médecin-vétérinaire attaché au Laboratoire.

Nous exposerons la partie thérapeutique de notre travail dans une étude spéciale. Ici, nous ne nous occuperons que des espèces de trypanosomes rencontrées durant cette période, soit à Stanleyville-même, soit dans un certain nombre d'autres localités de la Province Orientale.

Les trypanosomoses animales du Bas-Congo ont été bien étudiées par les divers savants du Laboratoire de Léopoldville : Broden, Rodhain, Van den Branden et Van Hoof. Les trypanosomoses animales du Katanga nous sont également bien connues, gràce aux études de la Mission Scientifique du Katanga (Rodhain et collaborateurs), de Walravens et de Schwetz. En ce qui concerne la Province Orientale, nous avons quelques renseignements sur la trypanosomose animale de l'Uélé, toujours gràce à l'infatigable Rodhain. Mais c'est tout. Et disons en passant que Rodhain a trouvé, sur 93 bovidés examinés dans diverses localités de l'Uélé : 11 fois $T$. dimorphon (congolense) et 8 fois $T$. cazalboui (vivax). Il n'a par contre rien trouvé chez les moutons, ni chez les chèvres, ni chez les équidés (ânes et chevaux) examinés, il est 'vrai, en trop petit nombre.

Il eût été injuste de ne pas mentionner, en parlant des trypanosomoses du Congo, les travaux classiques de Dutton et Todd, ces pionniers scientifiques de la médecine au Congo belge. En ce qui concerne les trypanosomoses animales, elles furent déjà étudiées

Annales de Parasitologie, $\mathrm{T}$. IX, $\mathrm{N}^{\circ} 5 .-1^{\mathrm{er}}$ septembre 1931 , p. 392-422. 
par ces deux savants en 1907, à l'époque héroïque, peut-on dire, de la médecine tropicale. Dutton et Todd ont décrit les trypanosomoses animales trouvées par eux dans diverses parties du Congo et, entre autres, dans diverses localités de la Province orientale dont nous nous occupons dans notre étude. Si grands savants les pionniers soient-ils, ils ne peuvent évidemment pas ne pas commettre, à cause de la nouveauté, et de l'abondance des sujets à étudier, certaines erreurs dans les détails. Il appartient aux générations suivantes de corriger peu à peu ces erreurs.

Les divers trypanosomes pathogènes du bétail étaient considérés par Dutton et Todd comme une seule et même espèce polymorphe, décrite sous le nom de $T$. dimorphon. Il suffit de lire les travaux des deux savants précités pour se convaincre qu'ils avaient en réalité affaire à plusieurs espèces de trypanosomes et en tous cas à $T$. congolense et à $T$. vivax (et probablement aussi à $T$. uniforme).

Le nom de $T$. dimorphon fut done donné par Dutton et Todd à une infection mixte. Plus tard, le nom de $T$. dimorphon fut donné à une variété spéciale de $T$. congolense et encore plus tard il devint peu à peu le synonyme de $T$. congolense. D'où une grande confusion dans laquelle il n'est pas facile de se débrouiller.

Abstraction faite de quelques rares équidés et de quelques rares animaux sauvages (gibier), nous avons eu affaire à trois groupes d'animaux domestiques : aux bovidés, aux ovidés et aux suidés. Ces trois catégories peuvent encore être subdivisées en trois groupes :

1. Animaux domestiques qui se trouvaient à Stanleyville depuis déjà plusieurs années ;

2. D'autres, que nous examinions à leur arrivée à Stanleyville : de l'Uélé, de l'Ituri, du Kivu ou du Ruanda ;

3. Enfin, des bêtes examinées à frais, sur place, dans diverses localités de l'Uélé (par M. Storck) et dont les gouttes épaisses et les frottis étalés furent ensuite étudiès au Laboratoire de Stanleyville.

Nous ne nous occuperons pas ici de l'aspect étiologique, ou épizootologique, de nos diverses trypanosomoses. Cet aspect de la question est si compliqué et si obscur qu'il mérite une investigation spéciale. Dans l'Uélé, la Glossina palpalis est quasi-ubiquiste autour des cours d'eau et la $G$. fusca y existe également. Dans toute la vaste forêt qui sépare le Kivu de Stanleyville, il existe trois espèces de giossines : G. palpalis, G. fusca et G. tabaniformis. On s'explique done facilement les trypanosomoses animales de l'Uélé et celles des bêtes venant d'arriver à Stanleyville, soit de l'Uélé, 
soit du Kivu. Mais nous avons trouvé des trypanosomoses chez des bovidés se trouvant déjà à Stanleyville depuis plusieurs années. Nous avons mème trouvé des trypanosomes chez des veaux nés à Stanleyville, c'est-à-dire dans les fermes environnantes. Or, si nous avons capturé dans les environs de Stanleyville les trois espèces de glossines qui existent dans toute cette région : G. palpalis, G. fusca et G. tabaniformis, toutes ces tsé-tsé sont si rares dans les environs de la ville qu'il est vraiment difficile de leur attribuer les vraies poussées épizoctiques de trypanosomes observées dans quelques fermes. Sans entrer ici dans de longues considérations, nous nous voyons obligés d'expliquer ces poussées par l'intervention d'insectes hématophages, autres que les glossines, dont le rôle consisterait à transmettre mécaniquement l'infection introduite, ou plutôt transmise, précédemment par les tsé-tsé.

Ceci dit, passons au sujet proprement dit de notre présente étude. Comme nous n'avons pas toujours trouvé les mêmes trypanosomes chez les trois groupes d'animaux domestiques, nous nous occuperons ici séparément des bovidés et des ovidés. Les divers trypanosomes trouvés chez les suidés sont exposés dans une étude spéciale.

\section{I. - Bovidés}

A. Bovidés se trouvant à Stanleyville ou dans ses environs depuis plusieurs années

\begin{tabular}{|c|c|c|c|c|}
\hline Troupeaux & 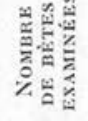 & 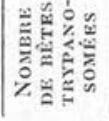 & $\begin{array}{c}\text { EsPĖcE } \\
\text { DE } \\
\text { TRYPANOSOMES }\end{array}$ & OBSERVATIONS \\
\hline $\begin{array}{l}\text { 1. Simi-Simi....... } \\
\text { 2. IJe Kangulu...... } \\
\text { 3. Kibibi.......... } \\
\text { 4. Sabeti .......... } \\
\text { 5. Ecole des Frères.. } \\
\text { 6. Mission catholique } \\
\text { 7. Tshopo......... } \\
\text { 8. Station .......... } \\
\text { 9. Lula............ } \\
\text { Total...... }\end{array}$ & $\begin{array}{r}28 \\
23 \\
454\end{array}$ & $\begin{array}{r}6 \\
8 \\
45 \\
\\
9 \\
0 \\
0 \\
7\end{array}$ & $\begin{array}{l}\text { T. congolense } \\
\text { T. congolense } \\
\text { T. vivax } \\
\text { T. uniforme } \\
\text { T. vivax } \\
\quad- \\
\quad- \\
\text { T. congolense } \\
\text { T. vivax } \\
\text { T. uniforme } \\
\quad- \\
\quad-\end{array}$ & $\begin{array}{l}\text { A } 7 \mathrm{k}^{\mathrm{ms}} \text { de la ville } \\
\text { A } 11 \mathrm{k}^{\mathrm{ms}} \text { de la ville } \\
\text { A } 7 \mathrm{k}^{\mathrm{m}} \text { de la ville } \\
\text { A } 3 \mathrm{k}^{\mathrm{ms}} \text { de la ville } \\
\text { En ville mème } \\
\text { A } 6 \mathrm{k}^{\mathrm{ms}} \text { de la vitle } \\
\text { A } 3 \mathrm{k}^{\mathrm{ms}} \text { de la ville } \\
\text { En ville même } \\
\text { A } 8 \mathrm{k}^{\text {ms }} \text { de la ville }\end{array}$ \\
\hline
\end{tabular}


B. Bovidés venant d'arriver à Stanleyville

\begin{tabular}{|c|c|c|c|c|}
\hline Troupeaux & 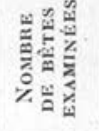 & 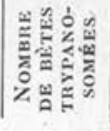 & $\begin{array}{c}\text { EsPÈcE } \\
\text { L.E } \\
\text { TrYPanosomes }\end{array}$ & Oaservations \\
\hline 1. Troupeau C..... & 32 & 20 & $\begin{array}{l}T . \text { congolense } \\
T \text {. vivax }\end{array}$ & de l'Uélé \\
\hline 2. Troupeau St..... & 29 & 17 & - & - \\
\hline 3. Troupeau Etat... & 156 & 156 & $\begin{array}{l}\text { T. congolense } \\
T \text {. vivax } \\
\text { T. uniforme }\end{array}$ & du Kivu \\
\hline 4. Troupeau Mat.... & 64 & 56 & - & du Ruanda \\
\hline 5. Troupeau Dili.... & 77 & 17 & $\begin{array}{l}10 T \text {. congolense } \\
7 T \text {. vivax }\end{array}$ & de l'Uélé \\
\hline 6. Troup. Lugwaret. & 33 & 2 & $\begin{array}{l}1 T . \text { congolense } \\
\text { 1. } T \text {. vivax }\end{array}$ & de l'Uélé \\
\hline 7. Troupeau Api.... & 22 & 1 & T. congolense & de l'Uélé \\
\hline 8. Troupeau Buta... & 20 & 14 & $\begin{array}{l}\text { T. congolense } \\
T \text {. vivax }\end{array}$ & de l'Uélé \\
\hline Total....... & 433 & 284 & & \\
\hline
\end{tabular}

Nous devons toutefois dire que pour certains troupeaux nous ne pouvons donner le chiffre absolument exact des bêtes trouvées trypanosomées, parce que plusieurs troupeaux furent examinés à plusieurs reprises avec un résultat différent à chaque examen. C'est ainsi qu'au premier examen du troupeau de la Kibibi (A 3), à la fin de 1927, nous n'avons trouvé que deux bêtes trypanosomées dont une avec de rares $T$. vivax et la deuxième, avec de rarissimes $T$. theileri. Mais au réexamen du même troupeau, une année plus tard, au début de 1928, nous avons trouvé un grand nombre de bêtes trypanosomées ( 45 sur 104, chiffre indiqué dans le tableau). Dans un autre grand troupeau (celui de l'Etat B. 3), le chiffre exact de bêtes trypanosomées est discutable poir la raison suivante. A l'examen de ce troupeau, dès son arrivée à Stanleyville, à la fin d'avril 1928, nous n'avons trouvé que 72 bêtes trypanosomées sur 156. Mais au deuxième examen de ce troupeau, en juillet, nous trouvàmes de nouvelles infections et, en septembre, lors du troisième examen, il ne restait plus une seule bête indemne. Devionsnous, dans ces conditions, donner le chiffre de 72 sur 156 ou celui de 156 sur 156 ? Quant aux autres troupeaux, nous avons indiqué le nombre de bêtes trypanosomées trouvées au premier examen, quoique à chaque réexamen de plusieurs troupeaux, après le trai- 
tement, nous trouvions parfois de nouvelles infections. Parmi ces dernières, il s'agissait parfois certainement de bêtes malades depuis longtemps, mais dont les trypanosomes n'avaient pas été vus lors d'un examen précédent à cause de la rareté momentanée des parasites.

Quoi qu'il en soit, il résulte du double tableau donné ci-dessus que nous avons trouvé une forte proportion de trypanosomés parmi les bovidés examinés à Stanleyville et que cette proportion était particulièrement grande parmi les bêtes qui venaient d'arriver. Ce qui se comprend d'ailleurs. N'ont survécu à Stanleyville que les bêtes particulièrement résistantes. Il s'agissait évidemment d'une sélection naturelle. Le sort ultérieur des troupeaux récemment arrivés, qui furent littéralement décimés, malgré un traitement varié et prolongé, est une confirmation de notre explication.

Voici à présent un relevé des bovidés examinés sur place, dans diverses localités de l'Uélé :

\section{Bovidés examinés dans l'Uélé}

\begin{tabular}{|c|c|c|c|c|}
\hline LocıLtrés & 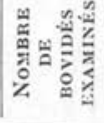 & 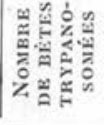 & $\begin{array}{c}\text { ESPÈCES } \\
\text { DE } \\
\text { TRYPANOSOMES }\end{array}$ & OBSERVATIONS \\
\hline 1. Ibembo... $\ldots \ldots$ & 28 & 5 & $\begin{array}{l}4 T \text {. congolense } \\
1 T \text {. vivax }\end{array}$ & \\
\hline 2. Buta............ & 130 & 0 & & \\
\hline 3. Titule .......... & 19 & 0 & & \\
\hline 4. Api............ & 85 & 0 & & \\
\hline 5. Bambesa ........ & 17 & 17 & $\begin{array}{l}13 \text { T. congolense } \\
3 T \text {. vivax } \\
1 \text { ? mixte }\end{array}$ & \\
\hline 6. Dembia.......... & 15 & 2 & $2 T$.congolense & \\
\hline 7. Aketi............ & 8 & 2 & $2 T$. congolense & \\
\hline 8. Dingila.......... & 21 & 1 & T. Iheileri & 1 seul trypanosome \\
\hline 9. Dili $\ldots \ldots \ldots \ldots \ldots$ & 92 & 23 & $\begin{array}{l}4 T \text {. congolense } \\
17 T \text {. vivax } \\
2 \text { mixtes }\end{array}$ & \\
\hline 10. Amadi........... & 70 & 2 & $2 T$. vivax & \\
\hline 11. Niangara ........ & 213 & 4 & T. vivax & \\
\hline 12. Dugu............ & 113 & 5 & $\begin{array}{l}1 T . \text { theileri } \\
2 T \text {. congolense } \\
2 T . \text { vivax }\end{array}$ & Plusieurs trypanosomes \\
\hline 13. Gangala ......... & 35 & 1 & T. vivax & \\
\hline Total ........ & 846 & 62 & & \\
\hline
\end{tabular}




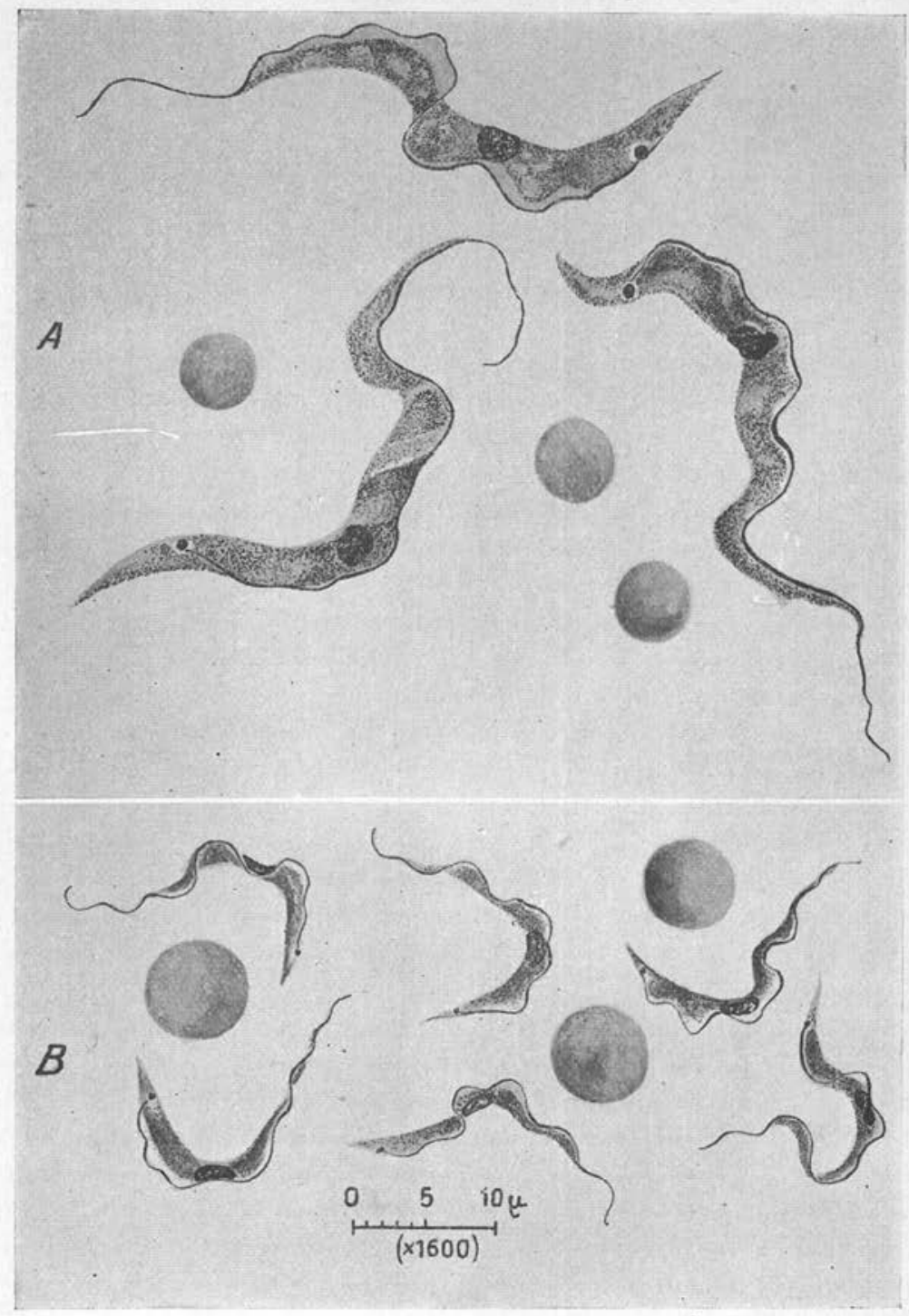

FIG 1.

A. Trypanosoma theileri. Taureau de Dungu (Uélé)

B. Trypanosoma brucei. Chien (!ac Albert). 
Les espèces de trypanosomes trouvés chez les bovidés de Stanleyville et de l'Uélé. Leur détermination et classification (fig. 1 à 6). - Disons de suite que, abstraction faite des trois bovidés à $T$. theileri, qui n'est d'ailleurs pas considéré comme pathogène, nous n'avons vu, chez nos centaines de bovidés, que deux groupes de trypanosomes : $T$. congolense et $T$. vivax, le premier beaucoup plus fréquent que le second.

On remarquera que, pour la plupart des troupeaux de Stanleyville, nous nous bornons à indiquer les espèces des trypanosomes trouvés, sans donner leur proportion relative. On remarquera également que nous signalons la présence de $T$. uniforme mais que nous ne mentionnons pas une seule fois la présence de $T$. dimorphon. Tout cela nécessite quelques éclaircissements.

Nous avions tâché de déterminer chaque fois le trypanosome auquel nous avions affaire, mais nous nous sommes vite aperçus que notre détermination était parfois - et même souvent - hasardée et même erronée.

La première grande difficulté pour la détermination exacte de nos trypanosomes provenait de la fréquence d'infections mixtes. C'est ainsi que nous avons trouvé chez de nombreuses bêtes, après le traitement, des $T$. congolense, quoique précédemment, avant le traitement, nous n'eussions vu que des $T$. vivax (ou des $T$. uniforme). C'est ainsi qu'actuellement, au moment où nous écrivons ces lignes, il n'existe plus, dans le vestige des trois grands troupeaux de Stanleyville, que $T$. congolense. Or, chez un certain nombre de ces bêtes, nous avions trouvé précédemment une infection mixte ; chez d'autres, T. congolense et chez d'autres enfin, T. vivax ou $T$. uniforme.

Ensuite, le $T$. congolense de nos bètes était rarement le petit $T$. congolense typique et classique. Dans la plupart des cas, il s'agissait d'un trypanosome plus long, avec parfois un rudiment de flagelle libre, bref un trypanosome que beaucoup d'auteurs décrivaient - et décrivent encore - comme $T$. dimorphon. D’autre part, dans la plupart des cas de nos $T$. vivax, il ne s'agissait pas du très long trypanosome avec un très long flagelle libre, bref du $T$. vivax classique et typique, mais d'un trypanosome beaucoup plus court et avec un flagelle libre également court.

De sorte que, s'il ne faut pas être grand spécialiste pour distinguer un $T$. vivax classique d'un $T$. congolense également classique, nous avouons nous être bien souvent trouvé très embarrassé devant certains de nos trypanosomes. Parce que, morphologiquement, il ne nous était pas toujours possible de décider si nous avions affaire 
à un « petit vivax» ou à un « grand congolense ». Certes, la distinction classique entre $T$. congolense et $T$. vivax consiste en l'ab-

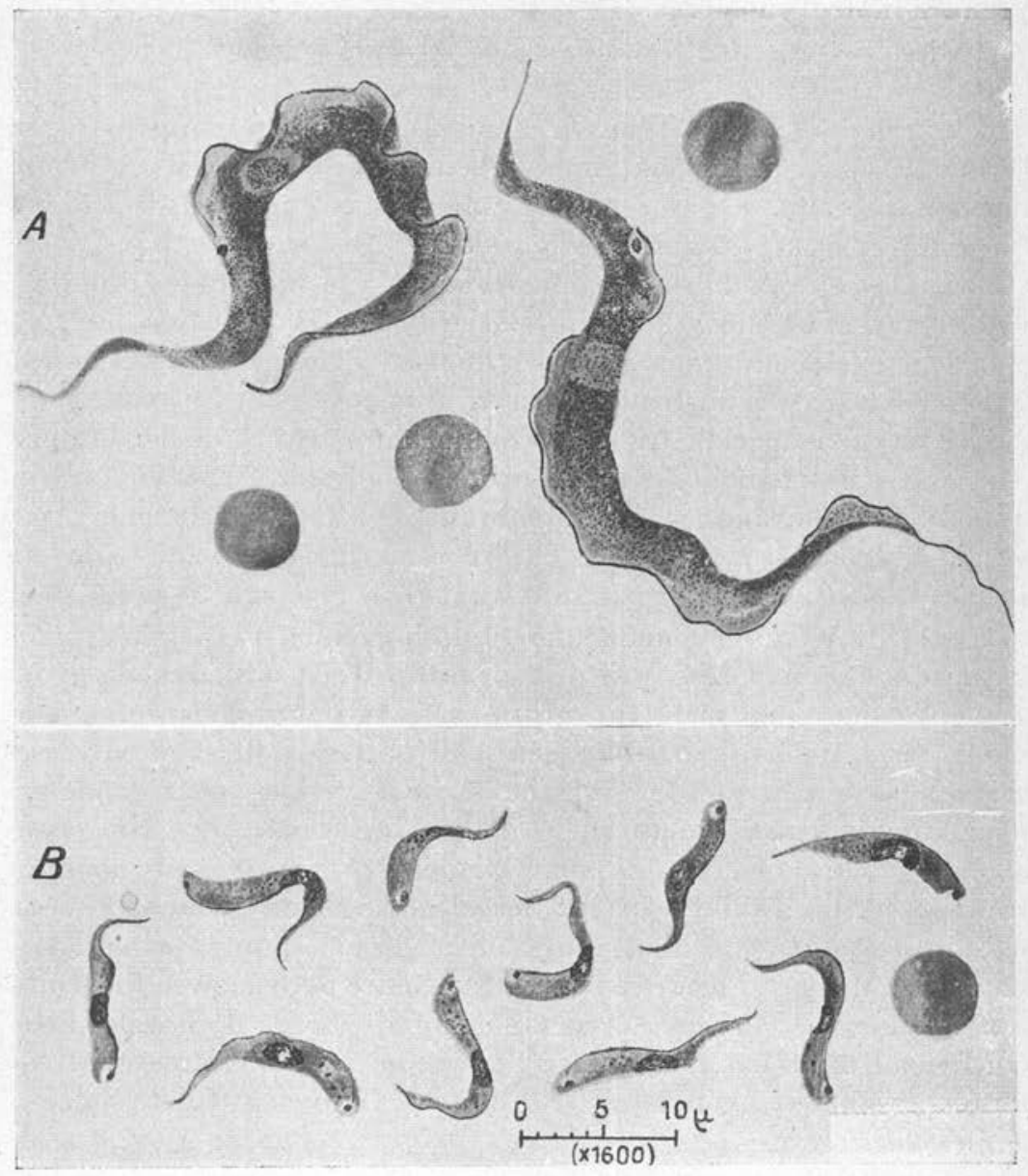

FIG. 2

A. Trypanosoma tragelaphi (?). Antilope harnassée : Tragelaphus sp. B. Trypanosoma congolense. Même antilope.

sence ou en la présence d'un flagelle libre, mais cette distinction n'est ni absolue, ni même constante. Beaucoup dépend de la coloration et du grossissement employé. De sorte que, parfois, on est forcé d'avoir recours au mot si peu précis de "presque »: 
presque pas de flagelle libre, presque un petit flagelle libre... Si nous ajoutons que notre détermination devait quelquefois se baser sur de rarissimes trypanosomes, déformés ou en division, on comprendra notre embarras.

Evidemment, outre les différences morphologiques, parfois peu nettes, entre diverses espèces de trypanosomes, il existe encore des différences biologiques, mais le temps nous a manqué pour avoir recours à cette différenciation. D'ailleurs, quelques essais de transmission que nous exposerons brièvement plus loin, nous ont donné un résultat nul, plutôt même paradoxal. De sorte que nous avons été forcés de nous borner exclusivement à la morphologie de nos divers trypanosomes. Pour ne pas trancher la question un peu à la légère et pour nous entourer de toutes les garanties scientifiques nécessaires à un travail sérieux, nous avons eu recours à plusieurs grands spécialistes en trypanosomes. Nous devons remercier tout spécialement le grand protozoologiste C.-M. Wenyon, qui a bien voulu examiner nos nombreux frottis et nous aider, par ses avis éclairés, à nous débrouiller dans la classification de nos trypanosomes.

Il est admis actuellement que $T$. dimorphon n'est qu'un synonyme de $T$. congolense, qui a la priorité. Il est entendu aussi que $T$. congolense appartient au groupe des trypanosomes monomorphes. Mais, d'autre part, tous ceux qui ont travaillé dans diverses régions savent que toutes les souches de $T$. congolense ne sont pas morphologiquement identiques. En ce qui nous concerne, nous avons été un peu dérouté par certaines formes de $T$. congolense trouvées à Stanleyville, surtout après celle vue précédemment dans le Haut-Katanga. Là, au Katanga, il s'agissait d'un tout pètit trypanosome de 8 à $12 \mu$, à extrémité postérieure obtuse, avec le blépharoplaste accolé à la paroi et sans la moindre trace de flagelle libre. On n'avait donc pas la moindre hésitation. D'autant plus que chez toutes les bêtes examinées, il s'agissait d'une infection pure à T. congolense.

Mais ici, à Stanleyville. $T$. congolense est moins typique et, disons même, un peu moins monomorphe. Il s'agissait, en général, d'un trypanosome plus long et plus mince, atteignant jusque 16 et même $20 \mu$, et avec un blépharoplaste moins typiquement latéral. Quant au flagelle, on a parfois une certaine peine à décider s'il est accompagné d'un amincissement du protoplasme jusqu'au bout ou non, bref, s'il existe un rudiment de flagelle libre ou non. Dans la plupart des cas, c'est uniquement à cette forme mince et relativement longue de $T$. congolense que nous avions affaire, mais parfois on 


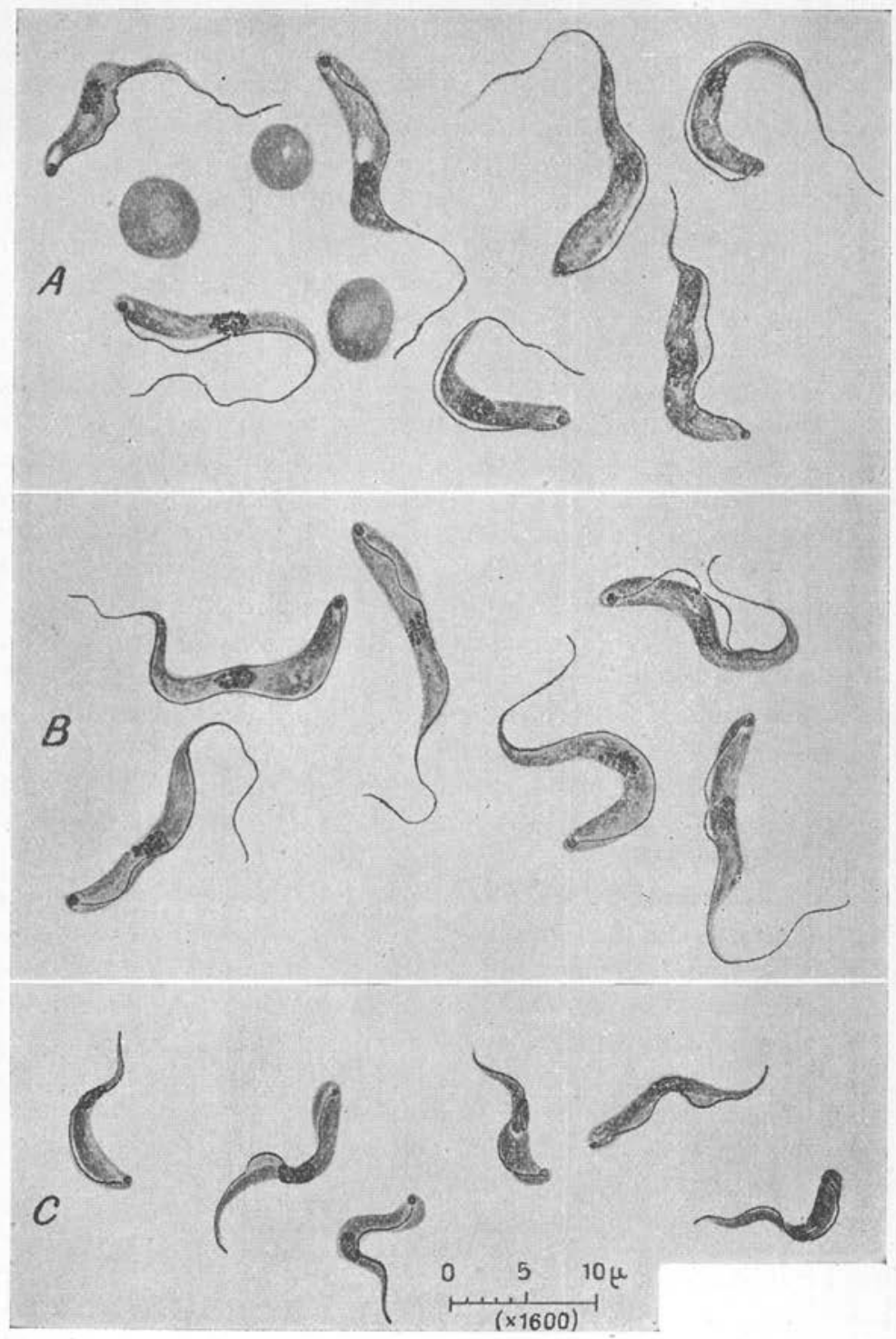

Fig 3

A. Trypanosoma vivax. Vache n 39 de Dili (Uélé).

B. Trypanosoma vivax. Vache ${ }^{\circ} 24$ de Dili (Uélé).

C. Trụpanosoma congolense. Vache n² 28 de Dili (Uélé). 
trouvait dans les préparations, à côté de cette forme, également des spécimens de la petite forme courte et trapue.

De sorte que s'il est logique de simplifier la classification des trypanosomes et de supprimer tous les synonymes de $T$. congolense (T. dimorphon, $T$. pecorum, $T$. nanum, etc.), il ne faut toutefois pas perdre de vue qu'il existe, morphologiquement du moins, deux variétés, ou deux formes, de T. congolense.

Il est également admis, d'autre part, que $T$. cazalboui (et $T$. angolense) ne sont que des synonymes de $T$. vivax. Mais si nous avons eu quelques difficultés à nous débrouiller dans nos $T$. congolense, nous avons eu encore plus de difficulté en ce qui concerne nos T. vivax. Parce que, à côté du vrai $T$. vivax typique, dont la longueur atteint jusque $25 \mu$, à extrémité postérieure arrondie ou un peu effilée, et avec un très long flagelle libre, nous avons eu affaire, dans les bêtes de Stanleyville, à un trypanosome morphologiquement identique au $T$. vivax, mais beaucoup plus court (de $15 \mu$ environ), et avec un flagelle libre également bien court. A frais, ce trypanosome, quoique de mouvements rapides, ne traverse toutefois pas le champ microscopique en flèche, comme le vrai T. vivax.

Notre "petit vivax» est donc, sans le moindre doute, T. uniforme. Chose curieuse : si, chez les bêtes de l'Uélé, nous n'avons vu que le vrai $T$. vivax, nous avons rencontré $T$. uniforme beaucoup plus fréquemment que T. vivax chez les bêtes de Stanleyville, aussi bien chez celles qui y étaient déjà depuis longtemps que chez celles qui venaient d'arriver du Kivu-Ruanda.

Ceci dit, on comprendra les difficultés que nous avons eues bien souvent à distinguer $T$. uniforme de la longue forme de $T$. congolense, surtout quand il s'agissait d'infection mixte et que les trypanosomes, port rares dans les frottis, étaient en division.

En résumé, nous dirons donc que chez les bovidés de Stanleyville nous avons trouvé trois espèces de trypanosomes pathogènes qui sont, par ordre de fréquence :

1. T. congolense.

2. T. uniforme.

3. T. vivax.

Chez les bovidés de l'Uélé, noứs n'avons trouvé que $T$. congolense et $T$. vivax.

Nous avons eu également l'occasion d'examiner le sang d'un certain nombre de bovidés du Kivu, soit sur place, soit surtout en frottis, envoyés au Laboratoire de Stanleyville. Il s'agissait, dans tous ces cas, de $T$. congolense. Mais il est évident que ces quelques examens ne peuvent exclure la présence d'autres trypanosomes chez les bovidés du Kivu. 


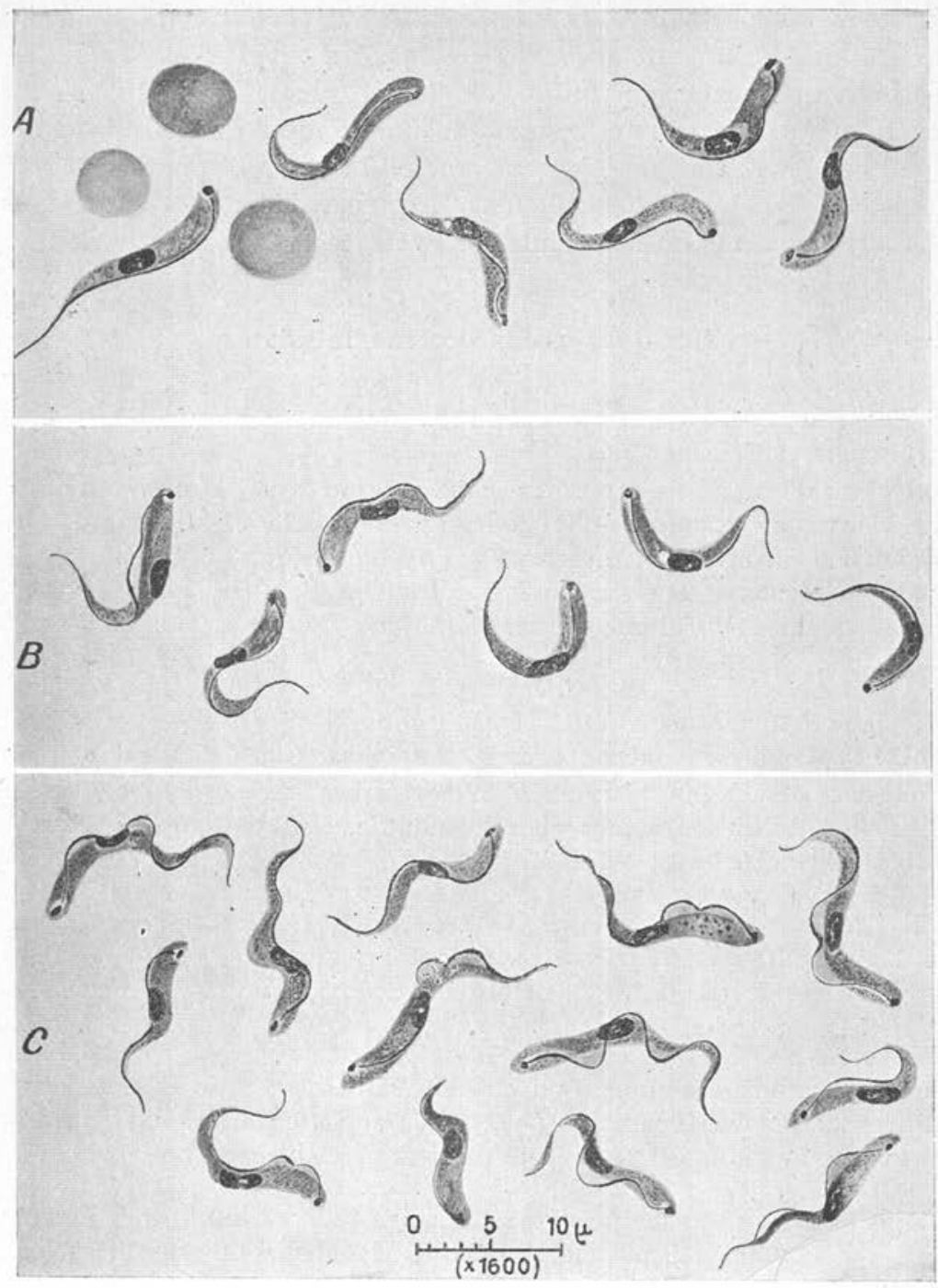

FIG. 4

A. Trypanosoma uniforme. Vache $\mathrm{n}^{\circ} 56$, Stanleyville.

B. Trypanosoma uniforme. Vache $\mathrm{n}^{\circ} 117$. Stanleyville.

C. Tryppanosoma brucei (?). Génisse, Stanleyville.

Nous n'avons vu qu'une seule fois T. brucei. Et encore, nous ne sommes pas sûr de notre détermination. 
Nous avons, par contre, trouvé trois fois T. theileri : une fois chez une bête de Stanleyville et deux fois chez des bovidés de l'Uélé. Chez une bête, une vache de Dungu, il y avait plusieurs trypanosomes dans le même frottis. A cela, nous devons ajouter un cas d'infection très intense à $T$. theileri, chez une vache du Kivu et provenant d'un troupeau infecté par $T$. congolense. Nous avons décrit ce cas peu ordinaire dans une note spéciale (Schwetz, 1930).

\section{Quelques essais de transmission}

I. Vache $n^{\circ} 111$. - Examinée le 20 septemble 1928. Infection mixte : $T$. uniforme et $T$. congolense.

Injection d'un $\mathrm{cm}^{3}$ de sang à un cobaye et de $2 \mathrm{~cm}^{3}$ à un mouton. Les deux bêtes sont examinées sept jours après, soit le 27 septembre. Chez le mouton on trouve de très rares $T$. congolense. (fig. $8 \mathrm{~B}$, mouton $\mathrm{n}^{\circ} 8$ ). Rien chez le cobaye. Il en est de même les 29 septembre, 3 et 12 octobre. Le mouton meurt le 12 novembre. Nombreux Cysticercus tenuicollis.

II. Vache $n^{\circ} 31$. - Examinée le 20 septembre 1928. T. congolense. Mème inoculation à un mouton et à un cobaye.

Le 27 septembre : mouton, rares $T$. congolense (fig. $8 \mathrm{C}$, mouton $\mathrm{n}^{\circ} 5$ ).

Cobaye : rien. Même résultat le 29 septembre. Le 3 octobre, on ne trouve plus de trypanosomes chez le mouton. Cobaye toujours négatif ; il en est de même le 12 octobre.

III. Vache $n^{\circ}$ 41. - Examinée le 19 octobre 1928. T. vivax. Inoculation à un mouton et à un cobaye. 25 octobre : mouton et cobaye négatifs ; il en est de même les 29 octobre et 8 novembre.

IV. Vache $n^{\circ}$ 23. - Examinée le 19 octobre. T. vivax et T. congolense. Inoculation à un mouton et à un cobaye. 25 octobre : mouton et cobaye négatifs. 29 octobre : mouton positif (T. congolense). Cobaye négatif. 8 novembre : mouton et cobaye positifs ( $T$. congolense).

V. Vache $n^{\circ}$ 131. - Examinée le 21 mars 1929. T. uniforme. Inoculation à un mouton et à un cobaye. Les deux bêtes sont examinées journellement. Le 31 mars ( $10^{\circ}$ jour), le mouton est positif, avec de très rares T. congolense. A partir de cette date on n'a plus trouvé de trypanosomes chez le mouton jusqu'au 23 avril, 34 jours après l'injection et date de sa mort. (Cysticercus tenuicollis). Le cobaye est resté négatif jusqu'au $73^{\circ}$ jour.

VI. Vean $n^{\circ} 40$ (Sabeti). - Examiné le 21 mars 1929. T. vivax. Inoculation à un mouton et à un cobaye. Examens quotidiens. Les deux bêtes restent négatives jusqu'au $73^{\circ}$ jour après la transmission. 
VII. Vache $n^{\circ} 70$. - Examinée le 21 mars 1929. T. congolense. Transmission à un mouton et à un cobaye. Examens quotidiens.

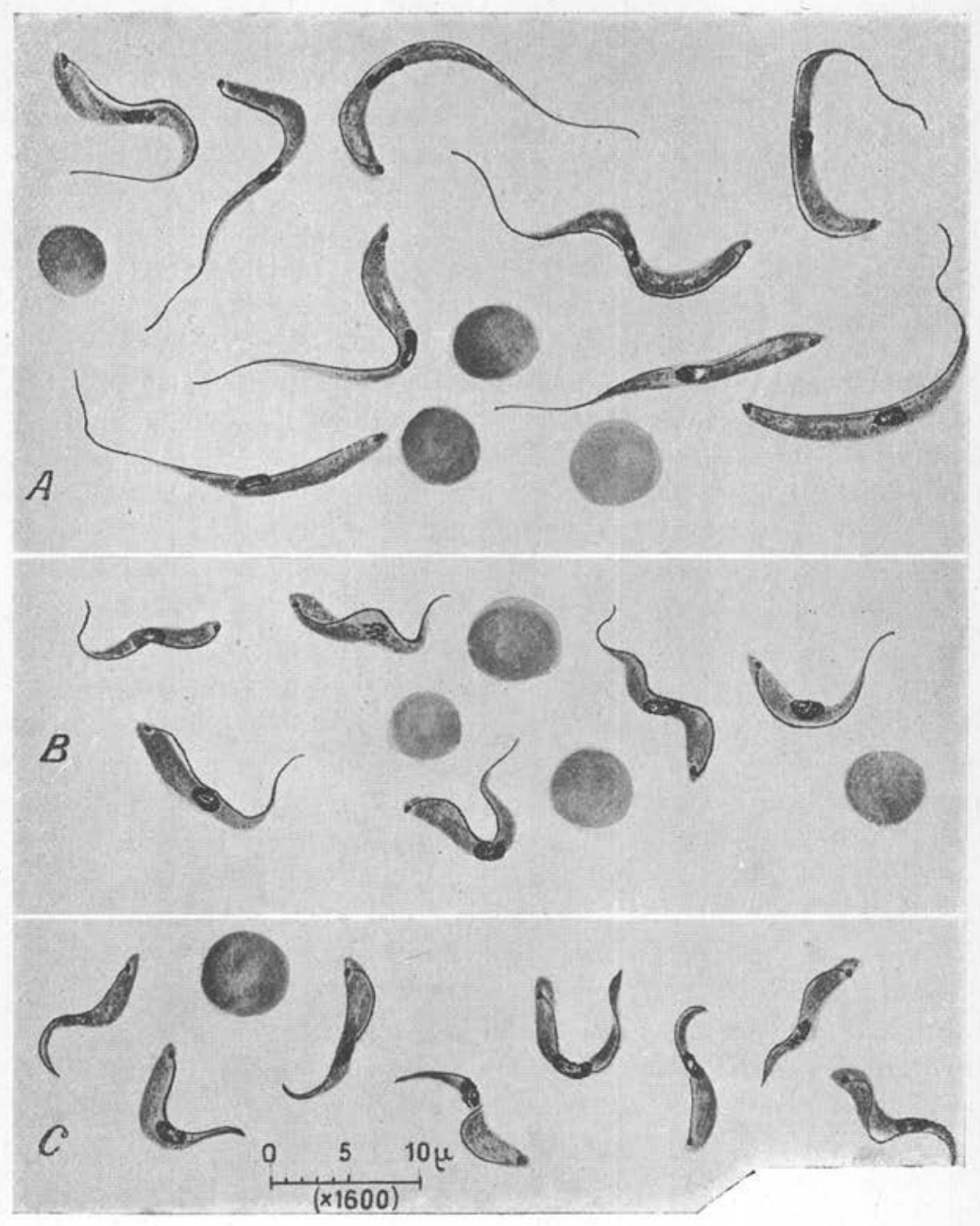

FIG, 5

A. Trypanosoma vivax. Génisse n ${ }^{\circ} 6$, Stanleyville.

B. Trypanosoma uniforme. Vache de Stanleyville.

C. Trypanosoma congolense. Vache de Lulenga (Kivu).

Le 29 mars ( $8^{\circ}$ jour), mouton positif : T. congolense.

Il reste positif jusqu'au $16^{\mathrm{e}}$ jour. Abattu in extremis (cysticerques). Le cobaye reste négatif jusqu'au $73^{\circ}$ jour. N'a plus été examiné ensuite. 
Après la mort du mouton, on injecte du sang de la même vache $\left(n^{\circ} 70\right)$ à un autre mouton qu'on trouve positif ( $T$. congolense) le $9^{\circ}$ jour. Il reste positif pendant vingt-cinq jours. Les trypanosomes sont tantôt rares et tantôt nombreux. Le mouton meurt le 22 mai. La transmission in extremis du mouton à un cobaye reste négative.

\section{Quelques considérations sur le résultat de nos transmissions. -} Ces transmissions furent faites dans un double but : d'abord confirmer notre détermination morphologique, et ensuite séparer les divers trypanosomes des infections mixtes. Mais, comme on vient de le voir, le résultat de presque toutes les transmissions fut non seulement nul, mais tout à fait paradoxal.

D'abord, les cobayes se montrèrent, dans toutes nos expériences, sauf une $\left(\mathrm{n}^{\circ} \mathrm{IV}\right)$, complètement réfractaires à l'infection. Si cela se comprend dans les expériences III, V et VI, où il s'agissait, comme il semblait résulter de l'examen microscopique, uniquement de $T$. vivax et de $T$. uniforme, le résultat est tout à fait incompréhensible dans les expériences I, II et VII, où il s'agissait de $T$. congolense.

Ensuite, nos moutons ne s'infectèrent que par $T$. congolense. Nous faisons abstraction de l'expérience III ( $T$. vivax), où le mouton n'a pu être examiné après le 20 jour. Admettons que dans les expériences $\mathrm{I}$ et $\mathrm{V}$, il $\mathrm{y}$ ait eu erreur de détermination microscopique et qu'en réalité il s'agissait dans ces deux expériences de $T$. congolense et non pas de $T$. uniforme. Nous avons effectivement expliqué plus haut les raisons pour lesquelles la distinction entre ces deux trypanosomes n'était pas toujours bien aisée. Une erreur a donc pu être commise. Mais dans l'expérience $\mathrm{n}^{\circ}$ VI, il s'agissait manifestement de $T$. vivax et de ce trypanosome seul. D'ailleurs, dans le petit troupeau auquel appartenait ce veau (A4, Sabeti), nous avons trouvé exclusivement $T$. vivax. Admettons qu'en réalité il s'agissait d'une infection mixte à $T$. vivax et à $T$. congolense, mais que les très rares $T$. congolense aient été « masqués » par de nombreux $T$. vivax. Mais alors, pourquoi n'avons-nous pas trouvé une infection mixte chez le mouton ? Pourquoi ce dernier ne s'est-il pas infecté de T. vivax ? De même pour l'expérience IV, où il s'agissait manifestement d'une infection mixte à $T$. vivax et à $T$. congolense? S'agissait-il d'une souche spéciale de $T$. congolense réfractaire aux cobayes ? Quant à nos moutons, ils devaient certainement être sensibles à l'infection par $T$. vivax, puisqu'ils provenaient d'un troupeau où nous avions constaté plusieurs cas d'infection par ce trypanosome. Mais il faut dire que chez les ovidés $T$. vivax (du moins celui de notre souche) semblait être très peu virulent et disparaissait à la longue de la circulation périphérique 

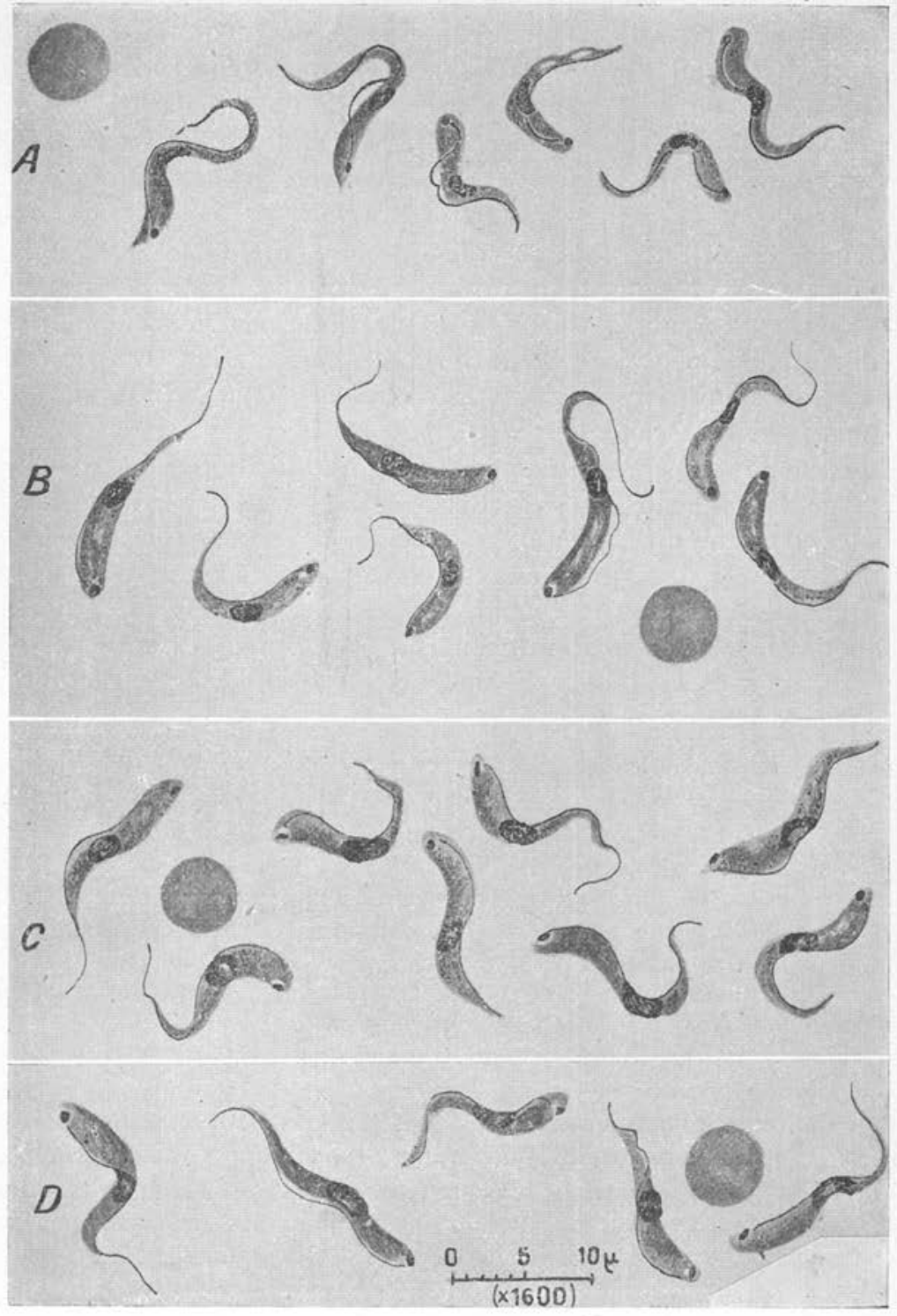

FIG. 6

A. Trypanosoma congolense. Vache $\mathrm{n}^{\circ} 1$ de Dili.

B. Taurillon Br. no 1. T. uniforme (et T. vivax ?).

C. Même bête, 7 jours après une injection d'émétique.

Trypanosoma uniforme et $T$. congolense.

D. Même bête deux jours plus tard : Trypanosoma uniforme et $T$. congolense. 
sans le moindre traitement. Il est donc possible que nos moutons d'expérience aient été immunisés (ou prémunis) contre $T$. vivax.

Quoi qu'il en soit, nous avons cru utile de signaler le résultat bizarre de nos expériences, bien qu'elles n'aient nullement facilité la détermination et la séparation de nos divers trypanosomes.

Deuxième série d'essais de transmission. - Une occasion unique s'est présentée à nous pour faire de nouveaux essais de transmission. Dans un troupeau, arrivé à Stanleyville des environs de Buta (Uélé) et fortement infecté de trypanosomes, nous trouvons plusieurs bêtes infectées par une seule espèce de trypanosome : soit $T$. vivax, soit $T$. congolense.

En même temps, nous trouvons chez un taurillon, seul survivant d'un petit troupeau parti du Kivu et-dont tous les animaux sont morts en route, une infection à $T$. uniforme et à Gonderia mutans (fig. VI B). Les deux premières bêtes ne sont pas traitées, en vue de la transmission, mais le taurillon reçoit entre temps une injection d'émétique. II reste stérile durant six jours, mais le septième jour, les trypanosomes réapparaissent. Cette fois-ci, il s'agit surtout de $T$. congolense (fig. VI C).

Le sang de chacune de ces trois bêtes est injecté à un mouton, un porc et un cobaye.

\section{Première bête. - Vache B. G. 7. T. vivax.}

Le cobaye et le porc restent négatifs pendant trente jours; le mouton meurt le onzième jour après l'inoculation, de cause inconnue, mais indemne de trypanosomes.

Deuxième bète. - B. G. 13. T. congolense.

Le cobaye reste négatif pendant trente jours.

Le mouton montre de rares $T$. congolense le septième jour après l'inoculation. Les jours suivants, il est tantôt positif et tantôt négatif. Meurt de pneumonie trente jours après l'inoculation (cysticercose).

Le porc devient positif le $14^{\mathrm{e}}$ jour ( $T$. congolense). Jusqu'au trentième jour, il est tantôt négatif et tantôt positif, mais les trypanosomes sont exirêmement rares et ne peuvent être décelés que dans les gouttes épaisses.

Troisième bête. - B. Br. $\mathrm{n}^{\circ}$ 1. T. congolense et T. uniforme.

Le cobaye reste négatif pendant trente jours.

Le mouton devient positif ( $T$. congolense) le $17^{\circ}$ jour et le reste jusqu'au $30^{\circ}$ jour. Meurt le trentième jour.

Le porc reste négatif jusqu'au trentième jour. 
Résumé. - Dans le premier cas ( $T$. vivax), l'échec de notre expérience est évidemment dû à la mort prématurée du mouton, seul animal sensible à l'infection par $T$. vivax.

Mais dans les deux autres cas, où il s'agissait de $T$. congolense, nous avons fait la même constatation bizarre que dans les essais précédent : tandis que les moutons et les porcs s'infectaient, les cobayes restaient stériles.

Dans le troisième cas, il s'agissait évidemment, malgré le résultat du premier examen, d'une infection mixte.

Chez les moutons, aussi bien chez ceux de Stanleyville et provenant du Kivu, que chez ceux de l'Uélé, nous avons retrouvé nos deux espèces de trypanosomes pathogènes: $T$. congolense et T. vivax. Nous ne croyons pas avoir trouvé chez les moutons T. uniforme. Nous avons par contre trouvé chez nos ovidés quatre fois T. brucei : chez trois moutons de l'Uélé et chez un mouton de Stanleyville, qui venait d'arriver du Kivu. Chez ce dernier, T. brucei était associé à $T$. vivax.

Quoique ne nous occupant, dans cette note, que de l'aspect morphologique des trypanosomes, nous croyons toutefois utile de signaler, en passant, la moindre virulence de ces mêmes trypanosomes chez les ovidés que chez les bovidés. Voici, en effet, le sort des huit moutons trypanosomés du troupeau A 1 du tableau:

1. Un des moutons à $T$. congolense (très nombreux trypanosomes dans le sang) meurt le jour même de l'examen.

2. Le mouton à infection mixte (également très nombreux trypanosomes dans le sang) meurt deux jours après l'examen.

3. Les six autres moutons sont laissés sans traitement pour voir ce qui adviendra. Un des trois à $T$. congolense meurt d'une plaie infectée. Réexaminés un mois plus tard, les cinq moutons sont positifs, mais les trypanosomes sont beaucoup moins nombreux qu'au premier examen. Réexaminés deux mois plus tard, les trois moutons à $T$. vivax sont négatifs, les deux à $T$. congolense sont toujours positifs. Même résultat à l'examen suivant, deux mois plus tard. Les moutons à $T$. congolense sont traités alors au tryparosan. Les trypanosomes disparaissent, mais ils sont retrouvés deux mois plus tard. Les trois moutons à $T$. vivax sont toujours négatifs.

Nous avons donc constaté une guérison spontanée d'une durée de plusieurs mois chez trois moutons infectés par T. vivax. T. congolense est évidemment plus résistant, même chez les ovidés, mais il semble quand mème être moins virulent chez les ovidés que chez les bovidés. 
II. OvidÉs (fig. 7-10)

\begin{tabular}{|c|c|c|c|}
\hline Troupeau et localité & $\begin{array}{l}\text { NoMBRE DE } \\
\text { MOUTONS } \\
\text { EXAMINÉS }\end{array}$ & $\begin{array}{l}\text { NOMBRE DE } \\
\text { BÊTES TRY- } \\
\text { PANOSOMÉES }\end{array}$ & EsPĖCE DE TRYPANOSOMES \\
\hline A. Stanleyville & & & \\
\hline 1. Troupeau Etat..... & 40 & 8 & $\begin{array}{l}\text { 1. } 4 T \text {. congolense } \\
\text { 2. } 3 T \text {. vivax } \\
\text { 3. } 1 \text { infection mixte }\end{array}$ \\
\hline 2. Troupeau Kibibi... & 6 & 0 & \\
\hline 3. Troupcau Mathot... & 207 & 29 & $\begin{array}{l}\text { 1. T. brucei }+T . \text { vivax } \\
\text { 2. } 18 T \text {. vivax } \\
\text { 3. } 7 \text { T. congolense } \\
\text { 4. } 3 \text { mixte }\end{array}$ \\
\hline 4. Troupeau Tshopo.. & 22 & 5 & T. vivax \\
\hline 5. Troupeau $\mathrm{Km} 20 \ldots$ & 28 & 22 & $\begin{array}{l}7 T \text {. vivax } \\
14 T \text {. congolense } \\
1 \text { infection mixte }\end{array}$ \\
\hline B. Uélé & & & \\
\hline 1. Ibembo ........... & 103 & 3 & $\begin{array}{l}1 T . \text { congolense } \\
1 T \text {. vivax } \\
1 T . \text { brucei }\end{array}$ \\
\hline 2. Aketi............. & 6 & 1 & T. congolense \\
\hline 3. Bambesa.......... & 6 & 3 & $\begin{array}{ll}2 & T . \text { brucei } \\
1 & T . \text { vivax }\end{array}$ \\
\hline 4. Dingila............ & 2 & 0 & \\
\hline 5. Amadi ............ & 28 & 0 & 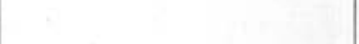 \\
\hline 6. Dungu $\ldots \ldots \ldots \ldots$ & 33 & 0 & . \\
\hline Total.......... & 481 & 71 & \\
\hline Capridés & & & \\
\hline 1. Ibembo........... & 69 & 1 & T. vivax \\
\hline 2. Bambesa.......... & 19 & 0 & \\
\hline 3. Niangara.......... & 41 & 0 & \\
\hline 4. Stanleyville (Mathot). & 7 & 1 & T. congolense \\
\hline 5. Stanleyville (Kibibi).. & 13 & 0 & \\
\hline Total........... & 149 & 2 & \\
\hline
\end{tabular}

III. - Autres animaux domestiques (ou domestiQués)

\section{A. Chevaux (Uélé)}

1. Buta ....................... 13

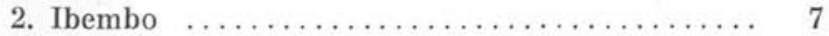

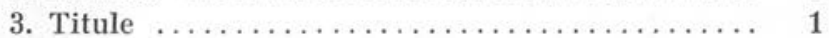

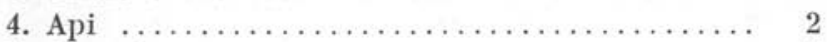

5. Gangala $\ldots \ldots \ldots \ldots \ldots \ldots \ldots \ldots \ldots \ldots \ldots \ldots \ldots \ldots \ldots$ 


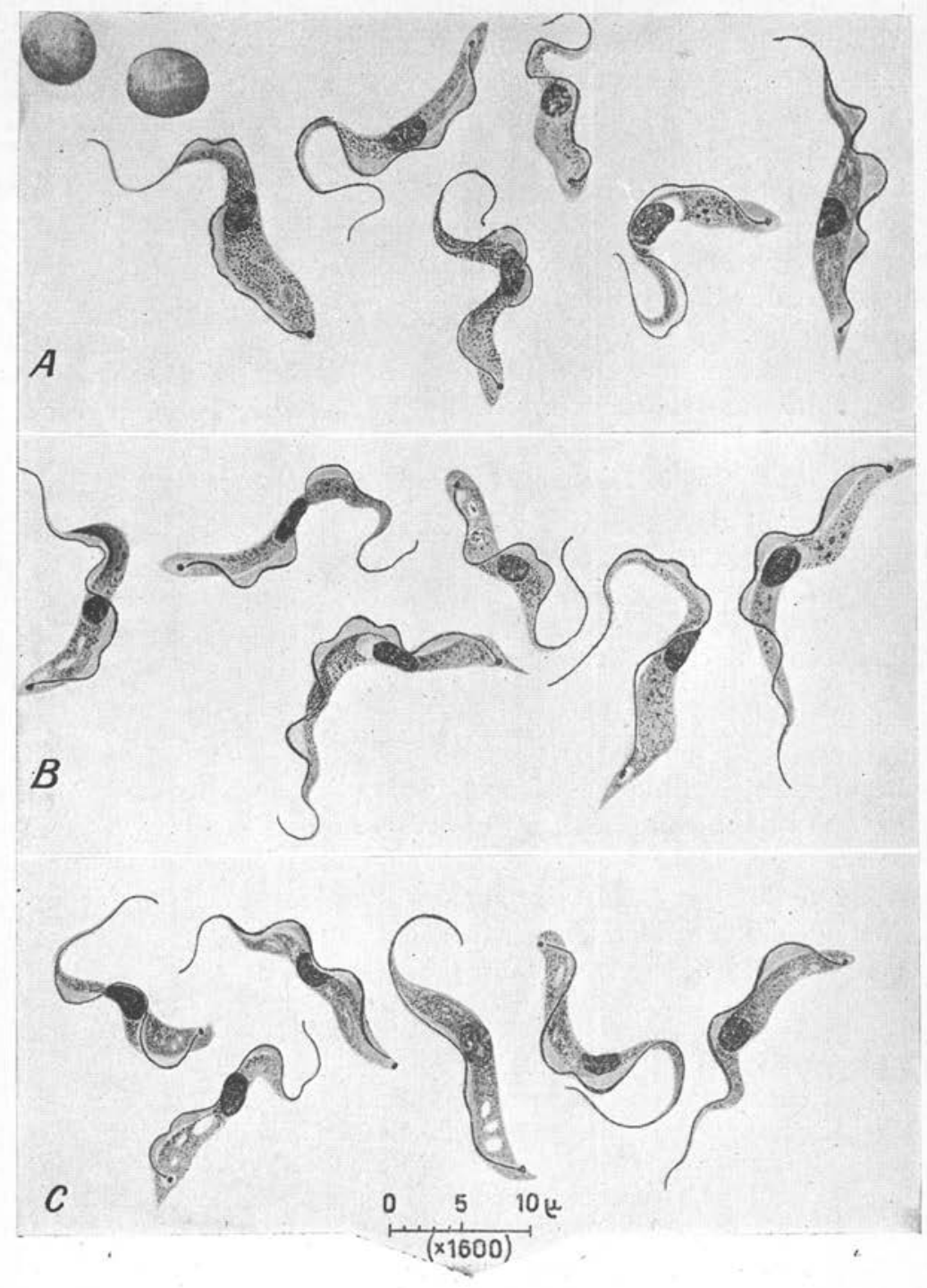

FIG. 7

A. Mouton n ${ }^{\circ} 1$, Bambesa (Uélê), Trypanosoma brucei.

B. Mouton no 2, Bambesa (Uélé), Trypanosoma brucei.

C. Mouton d'Ibembo (Uélé), Trypanosoma brucei.

Pas trouvé de trypanosomes. Nous avons trouvé trois chevaux parasités par $T$. congolense, dans les circonstances suivantes : 
Une de nos assistantes se trouvant à Kirundu (à $200 \mathrm{~km}$. au sud de Stanleyville), y prend quelques frottis d'un cheval maigre appartenant au chef de cette agglomération. Nous trouvons dans ces frottis de rares $T$. congolense. Nous ne connaissons pas le sort ultérieur du cheval.

Le deuxième cheval provient d'Api (Uélé). A son arrivée à Stanleyville, fin 1928, il est trouvé trypanosomé. Nous ne le perdons pas de vue, mais nous le retrouvons, fin 1929, dans une ferme à plusieurs kilomètres de Stanleyville. Il est maigre et toujours porteur de rares T. congolense.

Enfin, dans le sang de deux chevaux de Bili (Uélé), envoyés au Laboratoire, nous avons trouvé des $T$. congolense chez l'un d'eux.

\section{B. Eléphants domestiques (Uélé)}

1. Api $\ldots \ldots \ldots \ldots \ldots \ldots \ldots \ldots \ldots \ldots \ldots \ldots, 32$

2. Gangala $\ldots \ldots \ldots \ldots \ldots \ldots \ldots \ldots \ldots \ldots \ldots, 48$

80

Pas trouvé de trypanosomes.

$$
\text { IV. - Animaux sauvages (Gibier) }
$$

Le nombre d'animaux sauvages examinés par nous est vraiment trop insignifiant pour en tirer des conclusions. Dans les environs de Stanleyville, le gibier est rarissime. Pendant nos voyages, nous n'avons pu ni chasser ni faire chasser. Les frottis de sang reçus de divers côtés étaient, en général, très mal faits.

Voici ce que nous avons quand même trouvé :

1. 3 cochons rouges sauvages, environs de Stanleyville ............................ 0

2. 3 cochons rouges sauvages, Bas-Lomami...... $1 \mathrm{~T}$. congolense

3. 3 minuscules antilopes ("Boloko ", Raphoceros campestris?) environs de Stanleyville...... $1 \mathrm{~T}$. theileri

4. 1 antilope harnassée ( (Pongo », Tragelaphus sp.) origine exacte inconnue, trouvée à bord d'un a. T. congolense bateau à Stanleyville. - N. B. Les trypano- (très nombreux) somes, rares le 21 juin, deviennent très nom- $b$. T. tragelaphi breux le 30 juin, la veille de la mort de (Fig. $2 \mathrm{~A}$ ) l'antilope.

5. a. 6 Waterbuck (Cobus ellipsiprymmus) Rives du 0

b. 11 Cobus vardoni (Cob puku)....... Lac 1 T. congolense

c. 2 Ourebia hastata................

Total : $\overline{29}$

Total : 3 

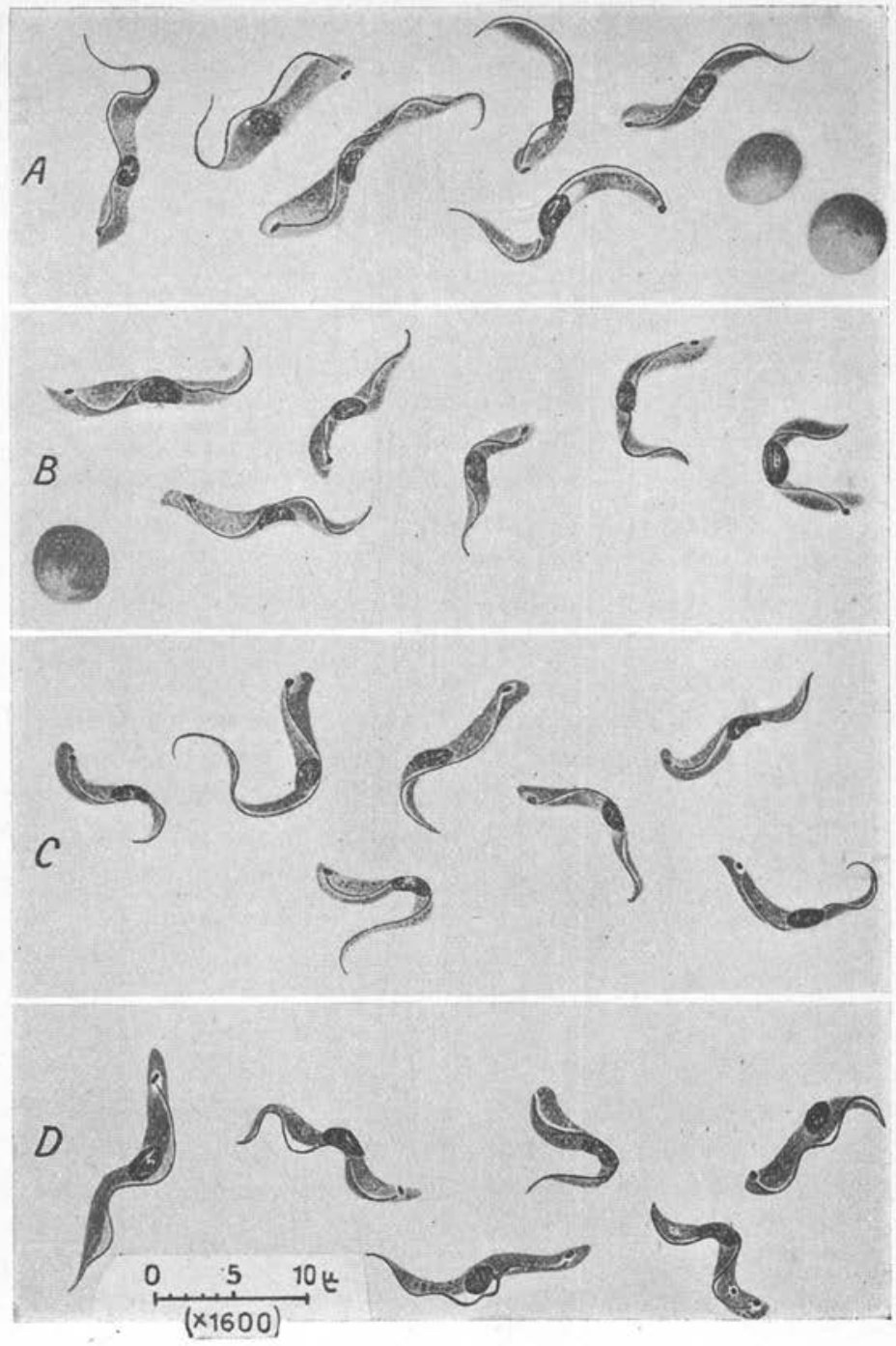

Fig. 8

A. Mouton no 15. Trypanosoma sp. (uniforme?)

B. Mouton n' 8. Trypanosoma congolense.

C. Mouton n ${ }^{\circ}$ 5. Trypanosoma congolense.

D. Mouton $\mathrm{n}^{\circ} 27$. Trypanosoma congolense. 


\section{Discussion}

Abstraction faite des suidés dont nous ne nous occupons pas dans cette étude, nous avons donc trouvé, chez les divers animaux domestiques de Stanleyville et de l'Uélé, quatre espèces de trypanosomes pathogènes :

1. T. congolense (bovidés, ovidés et équidés).

2. T. vivax (bovidés et ovidés).

3. T. uniforme (bovidés, et peut-être, ovidés).

4. T. brucei (ovidés, et peut-être, bovidés).

Nous avons, en outre, trouvé chez quelques bovidés $T$. theileri.

Chez les quelques rares bêtes sauvages examinées, nous avons trouvé $T$. congolense, $T$. theileri et $T$. tragelaphi (?)

$T$. congolense et $T$. vivax sont des espèces bien connues et communes. Leur présence au Congo Belge a été maintes fois signalée par plusieurs auteurs.

J'ai déjà insisté sur la diversité des formes ou des variétés de $T$. congolense. J'ai déjà dit plus haut que j'avais rarement trouvé ici les petites formes trapues typiques et que j'avais au contraire presque toujours eu affaire à des formes longues et minces et, bien souvent, avec un moignon de flagelle libre. C'est évidemment cette diversité morphologique de $T$. congolense qui est l'origine de la diversité des synonymes : $T$. pecorum, $T$. nanum et $T$. dimorphon.

En ce qui concerne T. vivax, on sait également que ce trypanosome, si typique soit-il, n'est pas absolument monomorphe. Il s'agit, dans la plupart des cas, d'un trypanosome à extrémité postérieure arrondie et à blépharoplaste terminal. Mais quelquefois on trouve des variétés à extrémité postérieure amincie et à blépharoplaste subterminal. Dans ces cas, le trypanosome est en général plus mince et plus long. Je ne donne pas ici les mensurations en microns, qui sont trop connues. Les écarts sont d'ailleurs trop grands pour donner des moyennes. Enfin, les figures jointes à cette étude, en diront plus long que les chiffres. C'est évidemment cette diversité morphologique du même trypanosome qui est l'origine des divers synonymes de $T$. vivax: $T$. cazalboui, $T$. capræ et $T$. angolense.

T. uniforme n'a pas encore été signalé au Congo Belge. Ce trypanosome, décrit par Bruce et ses collaborateurs dans l'Est africain, fut signalé ensuite dans les mêmes régions par divers autres auteurs. Curson signale, en passant, sa présence chez les bovidés du Zululand.

Mais qu'est-ce exactement que T. uniforme? En somme, la réponse à cette question est très simple : $T$. uniforme est un petit $T$. vivax. La seule différence de taille est-elle suffisante pour créer une espèce 


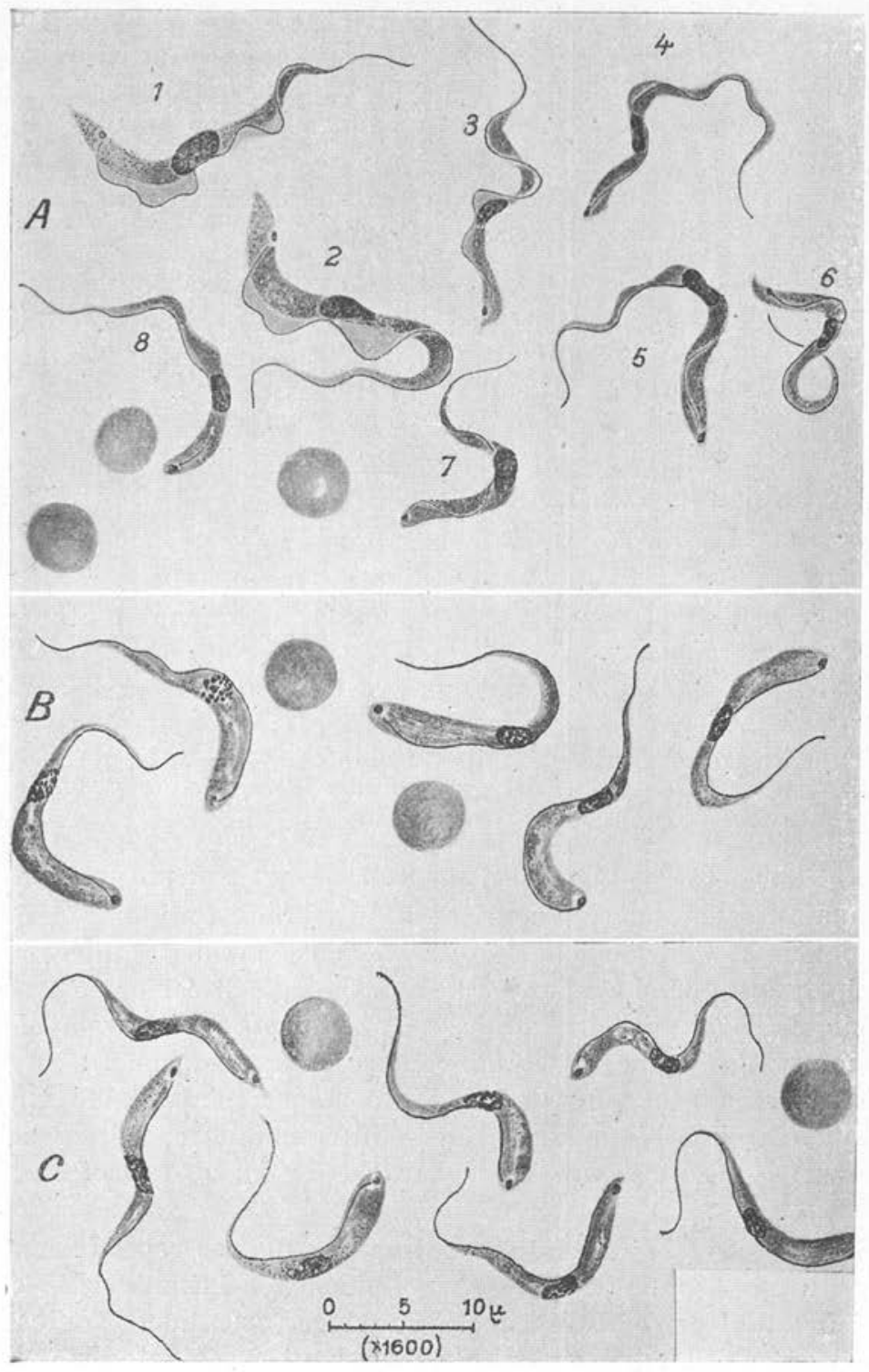

F1G. 9

A. Mouton $n^{\circ} 71$. Trypanosoma brucei (et T. vivax?)

B. Mouton Br. no 10. Trypanosoma vivax.

C. Mouton $\mathrm{Br}$. $\mathrm{n}^{\circ} 18$. Trypanosoma vivax. 
spéciale ? Nous avons bien vu que $T$. congolense comprend, suivant les cas, des trypanosomes longs et minces ou courts et trapus, et pourtant tout le monde admet à présent que dans tous ces cas, il s'agit de la même espèce. Pourquoi alors donner un nom spécial à une petite variété de $T$. vivax ?

Voici d'ailleurs un résumé de la description de T. uniforme donnée par Bruce et ses collaborateurs :

" De même que T. vivax, T. uniforme n'est pas pathogène pour les petits animaux de laboratoire (cobayes, rats, souris, chiens et singes). " « Les mouvements à l'état frais de T. uniforme sont intermédiaires entre ceux de $T$. vivax et ceux de $T$. pecorum ( $T$. congolense), c'est-à-äire qu'il se déplace dans le champ microscopique, mais n'en sort pas. T. uniforme a une longueur moyenne de $16 \mu$ (12-19) au lieu de $23 \mu 7$ (18-31) chez T. vivax.

Largeur: $1 \mu 5$ à $2 \mu 5(2-3 \mu$ chez $T$. vivax $)$.

Flagelle libre : 2 à $5 \mu$ ( 3 à $6 \mu$ chez $T$. vivax $)$.

Noyau : compact et central (fragmenté et occupant toute la largeur, chez T. vivax).

Partie post-nucléaire: pas aussi nettement rétrécie que dans T. vivax.

Membrane ondulante : étroite et peu développée (comme dans T. vivax).

En résumé : « $T$. uniforme ressemble à $T$. vivax par son aspect général, mais il s'en distingue par sa taille un peu plus petite. »

Nous verrons tout à l'heure qu'il n'est pas toujours facile de distinguer $T$. uniforme de $T$. vivax. Nous verrons d'ailleurs également qu'uniquement d'après la morphologie il est parfois même difficile de savoir si l'on a affaire à $T$. uniforme ou à $T$. congolense, du moins à la longue variété de ce dernier trypanosome.

$T$. brucei a beaucoup moins d'importance, n'ayant été vu que quelquefois chez les ovidés, soit en infection pure, soit associé à T. vivax. Nous ne sommes pas sùr d'avoir vu $T$. brucei chez les bovidés.

Passons à présent à l'interprétation des figures reproduisant les divers trypanosomes trouvés chez les bêtes de Stanleyville et de plusieurs autres endroits de la Province orientale. Toutes ces figures ont été dessinées par Mlle Droeshaut, assistante du Laboratoire.

La fig. $1 \mathrm{~A}$ représente trois spécimens de $T$. theileri, trouvé dans le sang d'un taureau de Dungu (Uélé).

La fig. 2 A représente deux spécimens d'un grand trypanosome trouvé (associé à T. congolense, fig. $2 \mathrm{~B}$ ) chez une antilope har- 
nassée : Tragelaphus sp. S'agit-il de $T$. theileri ou de $T$. tragelaphi ? Abstraction faite du noyau en bande chez le trypanosome de droite,
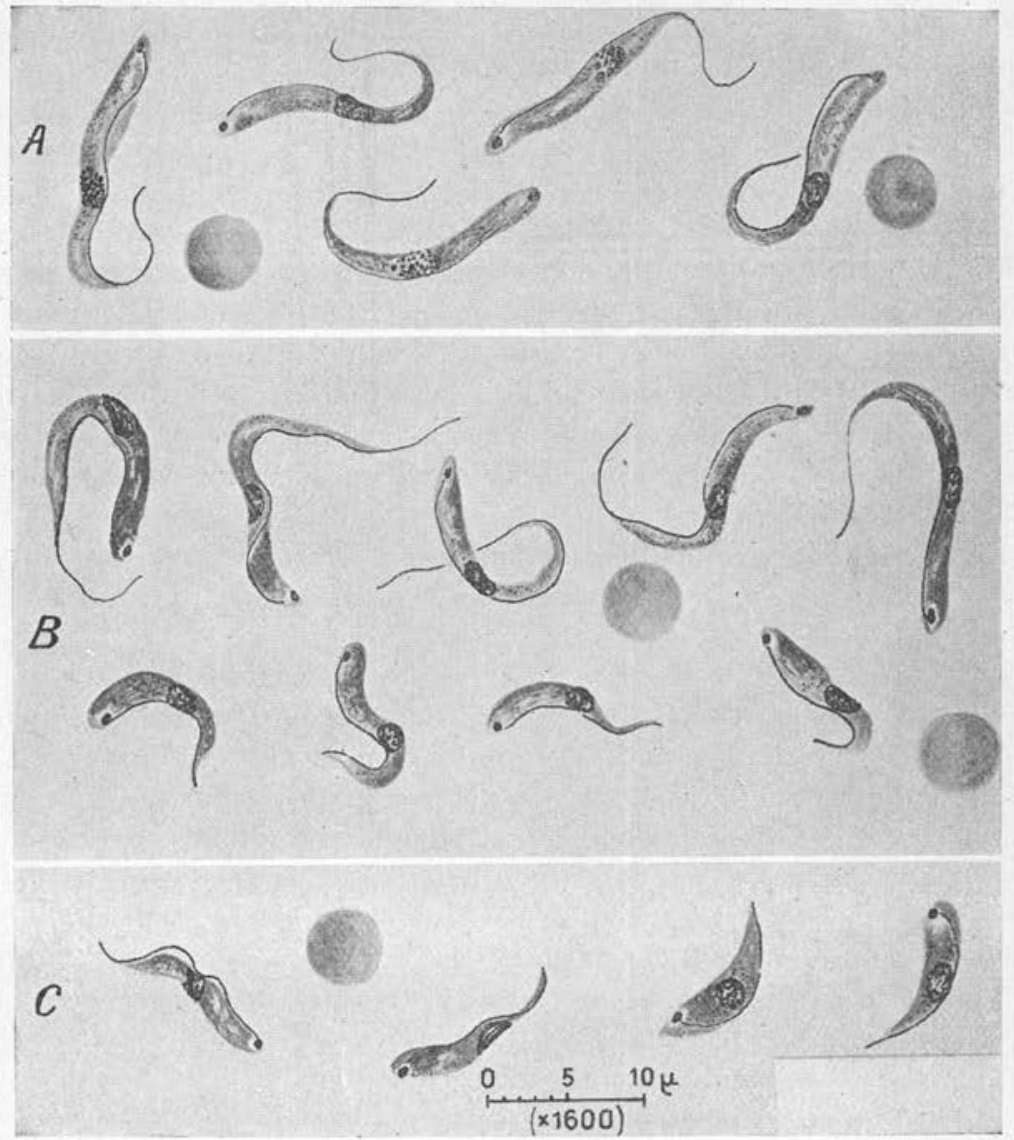

FIG. 10
A. Mouton $\mathrm{Br}$. No 13. Trypanosoma vivax.
B. Mouton $\mathrm{Br}$. No 7. Trypanosoma vivax et $T$. congolense.
C. Mouton $\mathrm{Br}$. $\mathrm{n}^{\circ}$ 19. Trypanosoma congolense.

les deux trypanosomes ressemblent plutôt à $T$. theileri qu'à $T$. tragelaphi.

La fig. $1 \mathrm{~B}$ représente plusieurs spécimens de $T$. brucei, provenant d'un chien de la région du Lac Albert. Elle servira de terme 
de comparaison avec certains trypanosomes trouvés chez des ovidés et même chez des bovidés.

La fig. 4 C représente des trypanosomes trouvés dans le sang d'une génisse de Stanleyville. De quelle espèce s'agit-il ici ? Plusieurs spécimens ressemblent un peu à $T$. brucei (membrane ondulante plissée et flagelle libre); d'autres, par contre, ressemblent à $T$. congolense. S'agit-il d'une infection mixte? Nous nous abstenons de trancher la question.

Les fig. $3 \mathrm{~A}$ et $3 \mathrm{~B}$ représentent $T$. vivax trouvé chez deux vaches de Dili (Uélé). La fig. 5 A représente $T$. vivax trouvé chez une vache de Stanleyville. On remarquera la grande différence entre les deux premières figures, d'une part, et la troisième, d'autre part. Les trypanosomes des vaches de Dili sont ceux décrits par Laveran et Mesnil sous le nom de T. cazalboui, tandis que ceux de la vache de Stanleyville ressemblent au trypanosome décrit par les mêmes auteurs, sous le nom de $T$. vivax (plus mince et extrémité postérieure effilée).

La fig. 3 C représente les trypanosomes trouvés chez une autre vache de Dili. Il s'agit évidemment de $T$. congolense (pas de flagelle libre).

Les fig. $4 \mathrm{~A}$ et $4 \mathrm{~B}$ représentent des trypanosomes trouvés chez deux vaches de Stanleyville. Il s'agit plus que probablement de $T$. uniforme. La fig. 5 B représente $T$. uniforme d'une vache de Stanleyville, et la fig. $5 \mathrm{C}, \mathrm{T}$. congolense d'une vache de Lulenga (Kivu). La fig. 6 A représente $T$. congolense d'une vache de Dili. Mais on remarquera qu'entre ces deux souches (ou variétés) de $T$. congolense, il existe une différence morphologique assez notable.

La fig. 6 B reproduit les trypanosomes trouvés (en mème temps qu'une forte infection à Gonderia mutans) chez un jeune taurillon arrivé récemment de Dili (Uélé) à Stanleyville. La plupart des trypanosomes ressemblent à $T$. uniforme, mais il y en a qui sont de vrais $T$. vivax! S'agit-il d'une infection mixte à $T$. uniforme et à $T$. vivax ? Ou s'agit-il tout simplement d'une variété de $T$. vivax, variété un peu moins monomorphe que d'habitude et comprenant à côté des grands spécimens habituels, d'autres, plus petits ?

Les fig. $6 \mathrm{C}$ et $6 \mathrm{D}$ représentent les trypanosomes trouvés chez le même taurillon, 7 jours et 9 jours plus tard, respectivement 7 et 9 jours après une injection d'émétique. Dans ces deux figures, nous trouvons, à côté de l'ancien trypanosome ( $T$. vivax-uniforme) un autre et notamment $T$. congolense.

Passons à présent aux trypanosomes tronvés chez nos divers moutons et qui sont reproduits dans les quatre dernières figures (fig. 7-10). 
La fig. 7 (A. B et C) reproduit des trypanosomes trouvés dans le sang de trois moutons : 2 de Bambesa et 1 d'Ibembo (Uélé). Il s'agit évidemment de $T$. brucei. Mais on remarquera que tous les trypanosomes sont beaucoup plus larges que ceux du chien (fig. $1 \mathrm{~B}$ ). Dans la fig. $7 \mathrm{~A}$, deux trypanosomes ressemblent singulièrement à $T$. vivax, de sorte qu'il s'agit probablement d'une infection mixte à $T$. brucei et à $T$. vivax. Il en est de même de la fig. $9 \mathrm{~A}$ (mouton de Stanleyville et provenant du Kivu) où il s'agit plus que probablement d'une infection mixte à $T$. brucei et à $T$. vivax. Mais chez ce dernier mouton, $T$. vivax a un aspect un peu différent et moins typique que T. vivax habituel (plus long et plus mince).

Par contre, dans les fig. 9 B et 9 C (deux moutons du Kivu qui venaient d'arriver à Stanleyville), il s'agit de $T$. vivax habituel et typique.

La fig. 8 reproduit des trypanosomes peu typiques trouvés chez quatre moutons de Stanleyville.

Dans $8 \mathrm{~A}$, l'espèce est vraiment difficilement déterminable. Presque tous les spécimens ont un flagelle libre bien net quoique peu long. Il s'agirait donc de T. uniforme. D'autre part, la plupart des spécimens ne sont pas « uniformes ». Le blépharoplaste est en outre, chez eux, plutôt latéral. De sorte que nous nous abstenons d'une détermination catégorique.

Dans B et C, il s'agit d'une infection expérimentale. Le mouton $n^{\circ} 8$ (B) a été infecté avec du sang d'une vache atteinte de $T$. congolense (et $T$. uniforme). Le mouton $\mathrm{n}^{\circ} 5$ (C) a été infecté avec du sang d'une vache atteinte de $T$. congolense. Chez ces deux moutons, nous avons trouvé $T$. congolense; mais la plupart des parasites appartiennent plutôt à la variété longue. Dans D, il s'agit d'une infection naturelle à $T$. congolense. Ici, nous trouvons les deux formes ou variétés : parasites courts et trapus et d'autres, plus longs et même avec un soupçon de flagelle libre.

La fig. 10 reproduit des trypanosomes trouvés chez trois moutons d'un petit troupeau qui venait d'arriver à Stanleyville, du Kivu.

Chez le mouton $\mathrm{n}^{\circ} 13(\mathrm{~A})$, nous avons trouvé une infection pure à $T$. vivax. Chez le mouton $\mathrm{n}^{\circ} 7(\mathrm{~B})$, nous avons trouvé une infection mixte à $T$. vivax et $T$. congolense. Chez le mouton $\mathrm{n}^{\circ} 19(\mathrm{C})$, nous avons trouvé une infection pure à $T$. congolense. Mais à còté des spécimens plutôt longs, comme chez le mouton précédent, nous avons trouvé ici également des formes trapues, très courtes et très larges.

Chez trois moutons du même petit troupeau, nous avons donc trouvé : une infection pure à $T$. congolense, une infection pure à 
T. vivax et une infection mixte. La détermination ne présentait dans ces trois cas aucune difficulté, les parasites ayant été trouvés en grand nombre et les spécimens étant bien typiques (abstraction faite de ceux en division que nous n'avons évidemment pas reproduits dans les figures). Au même petit troupeau appartenait le petit taurillon de la figure $6(\mathrm{~B}, \mathrm{C}, \mathrm{D})$, chez lequel nous avons trouvé, suivant les jours : T. uniforme (et $T$. vivax) et $T$. uniforme avec $T$. congolense.

\section{RÉSUMÉ}

Abstraction faite des suidés dont nous ne nous occupons pas dans cette étude, et dont nous avons décrit ailleurs une trypanosomose spéciale, nous avons trouvé chez les animaux domestiques de Stanleyville, animaux provenant du Kivu, du Ruanda, de l'Ituri et de l'Uélé, les trypanosomes suivants :

A. Ghez les bovidés: 1. T. congolense; 2. T. vivax; 3. T. uniforme ; 4. T:brucei; 5. T. theileri.

B. Chez les ovidés: 1. T. congolense; 2. T. vivax; 3. T. uniforme; 4. T. brucei.

C. Chez les équidés: $1 . T$. congolense.

D. Ghez le gibier (matériel insuffisant): 1. T. congolense; 2. T. theileri; 3. T. tragelaphi.

C'est $T$. congolense qui est l'espèce la plus commune et partant la plus importante. Viennent ensuite, par ordre de fréquence et d'importance, $T$. uniforme (chez les bovidés) et $T$. vivax (chez les ovidés). T. brucei n'a été trouvé que chez quelques ovidés, seul ou associé à $T$. vivax. Nous ne sommes pas sûrs d'avoir vu $T$. brucei chez les bovidés.

Dans la majorité des cas, il s'agissait d'une infection mixte à $T$. congolense et à $T$. vivax, circonstance qui rendait parfois bien difficile la détérmination exacte de nos trypanosomes. C'est cette fréquence de l'infection mixte qui est la cause de la description de tous ces trypanosomes sous le nom de $T$. dimorphon par Dutton et Todd eit de la confusion qui a longtemps régné dans la bibliographie. D'autre part, la détermination morphologique exacte de tous ces trypanosomes n'est pas toujours aisée.

Le $T$. congolense typique de Broden est plutôt extrêmement rare dans la Province Orientale et surtout à Stanleyville. Ici, il s'agit en général d'un $T$. congolense moins typique, c'est-à-dire de la forme mince et longue avec, parfois, un très court flagellè libre.

Le vrai $T$. vivax a été trouvé souvent chez les ovidés, mais bièn 
rarement chez les bovidés. Chez ces derniers, il s'agissait le plus souvent d'un petit $T$. vivax, c'est-à-dira de $T$. uniforme. Parfois, il était d'ailleurs difficile de dire s'il s'agissait de $T$. vivax ou de $T$. uniforme, soit parce que les trypanosomes trouvés étaient de taille intermédiaire, soit parce qu'il y en avait de taille différente. Et si, dans le deuxième cas, on pouvait admettre une infection mixte à $T$. vivax et à $T$. uniforme, la détermination restait douteuse dans le premigr cas. Les essais de transmission à d'autres animaux ne pouvaient en l'occurence être d'aucune utilité, la pathogénicité de ces deux trypanosomes étant la même. S'agit-il dẹ deux espèces différentes ou $T$. vivax et $T$. uniforme ne sont-ils que deux variétés de la même espèce, de même que les grandes et les petites formes de $T$. congolense? Ce ne sont que de longues observations et nouvelles rechęrches qui pourront trancher cette question, plus théorique d'ailleurs que pratique.

Les grandes formes de $T$. congolense, d'une part, et la présence de $T$. uniforme, d'autre part, nous ont fait parfois douter si nous avions affaire au groupe vivax ou au groupe congolense. C'était surtout le cas quand les trypanosomes étaient très rares dans les frottis et que la plupart des parasites étaient en division.

Dans les cas d'infection mixte, c'était en général $T$. vivax qui dominait et qui masquait par sa quantité les rares $T$. congolense. Mais quelques jours après une injection d'émétique, on voyait apparaître (ou plutôt réapparaître) les $T$. congolense, tandis que les $T$. vivax (ou $T$. uniforme) restaient invisibles.

Les quelques inoculations que nous avons tentées, aussi bien pour déterminer nos trypanosomes - dans les cas douteux - que pour séparer les diverses espèces dans les infections mixtes, nous ont donné des résultats négatifs et bien curieux.

A part une exception, les cobayes se sont montrés réfractaires à nos diverses souches de $T$. congolense; tandis que l'on trouvait des trypanosomes quelques jours après l'inoculation chez le mouton et mème chez le porc, les cobayes restaient négatifs. Les quelques essais de transmission de $T$. vivax (resp. T. uniforme) restèrent complètement négatifs : ou les moutons s'infectaient de $T$. congolense dans les infections mixtes, ou ils restaient négatifs dans les cas d'infection pure à $T$. vivax. Et pourtant $T$. vivax fut trouvé si souvent en infection naturelle chez d'autres moutons !

Il en résulte, ca qui est d'ailleurs connu, qu'à côté de l'espèce ou de la variété morphologique, la souche joue également un grand rôle dans la pathogénicité des trypanosomes. 


\section{BIBLIOGRAPHIE}

Bruce (D.), Hamerton (A.), Bateman (H.), Mackie (F.) et Lady Bruce. - Trypanosoma uniforme n. sp. Rep. sleeping sickness Commission Roy. Soc., $\mathrm{n}^{\circ} 11$, London, 1911.

Curson (H.). - Nagana in Zululand. 13 th and 14 th Rep. Director veter. res. Union South A frica, Pretoria, 1928.

Dutron (J.), Tood (Y) et Kinghorn (A.). - Cattle trypanosomiasis in the Congo free State. Ann. of trop. med and paras., I, 1907, p. 233-271.

KNeth (P.) et Du Toit (I'. J.). - Tropenkrankheiten der Haustiere in Mense, Handbuch der Tropenkrankheiten, VI, 1921, Leipzig.

Laveran et Mesnil - Trypanosomes et trypanosomiases. 2e édit., Paris, Masson, 1912.

Rodhain (J.). - Note sur les trypanoses et les piroplasmoses des grands animaux de l'Uélé. Bull. Soc. pathol. exot., IX, 1916, p. 95-109.

Schwetz 'J.). - Sur un cas d'infection intense par Trypanosoma theileri. Ann. de parasitologie, VIII, 1930 , p. $135 \cdot 139$.

Wenyon (C.-M.). - Pro'ozoo'ogy. London, 1926.

Laboratoire de Parasilologie de Slanleyville (Congo belye) 